\title{
Using Nonuniform Fiber to Generate Slow Light via SBS
}

\author{
Wenhai Li, ${ }^{1}$ Xiaoyi Bao,, ${ }^{1}$ V. P. Kalosha, ${ }^{1}$ Liang Chen,, ${ }^{1}$ and Ming-Jun Li ${ }^{2}$ \\ ${ }^{1}$ Department of Physics, Faculty of science, University of Ottawa, Ottawa, Ontario, Canada K1N 6N5 \\ ${ }^{2}$ Corning Incorporated, Corning, NY 14831, USA
}

Correspondence should be addressed to Xiaoyi Bao, xbao@uottawa.ca

Received 25 February 2008; Accepted 28 March 2008

Recommended by Wojtek J. Bock

The data pulse delay based on slow light induced by stimulated Brillouin scattering (SBS) in a nonuniform dispersion decreasing fiber (DDF) is demonstrated experimentally, and the distortions of data pulses at different beat frequencies are studied. We found that a delay exceeding a pulse width can be achieved at particular beat frequency, and the DDF has larger delay versus gain slope coefficient with much better output pulse quality than single-mode fiber.

Copyright ( $) 2008$ Wenhai Li et al. This is an open access article distributed under the Creative Commons Attribution License, which permits unrestricted use, distribution, and reproduction in any medium, provided the original work is properly cited.

\section{Introduction}

Slow light via stimulated Brillouin scattering (SBS) in optical fiber has been an active research area since 2005 [1-5]. The advantage of achieving slow light in fiber using SBS is the low threshold power, robustness, and simplicity of operation as it can be easily integrated with existing fiber system for optical signal processing, data buffers, and optical equalizers. The spectral bandwidth of the Brillouin scattering in a standard single mode fiber is about $30 \mathrm{MHz}[1,2]$, which is much narrower than the bandwidth of modern communication systems, that are utilizing GBytes/sec data streams. In order to solve this problem, various modulation schemes to broaden the Brillouin spectrum are proposed for the pump source (to slow the light) or the probe source (to advance the light) [3-6]. These schemes all suffer from considerable signal distortion due to maximum Brillouin gain at peak frequency of the pump wave because the beat frequency and phase-locking condition are only satisfied for the peak frequency of pump and probe wave, especially when the pulse trains are used, where intersymbol interference (ISI) poses another limitation [3]. The maximum pulse delay is limited by saturation of the pulse amplification via the Brillouin gain, which is a dispersion process. When a large delay is required, either high pump power or long fiber length is needed; hence the gain saturation at peak frequency is unavoidable. In other words, the high pump power leads to the power increase to the probe (data) which depletes the pump power. As a result, the Brillouin gain becomes location and frequency dependent, so does the amplified data signal (pulse), especially for pulse trains, where the rise and fall time could not be detected correctly due to the SBS slow-lightinduced distortion. Both saturation of the probe (signal) and depletion of the pump prevent SBS slow light in optical processing and as an optical buffer in fiber communications. Fortunately, both impairments can be mitigated by the nonuniform fiber as an SBS slow light generator based on distributed Brillouin frequency along the fiber length. Hence, the effective length for each Brillouin frequency is much shorter than the total fiber length in the pump modulation method [4-6], which creates an effective modification of the Brillouin frequency bandwidth in the fiber with fixed central frequency along the entire fiber length. With the use of a nonuniform fiber, we obtained a variable Brillouin frequency along the fiber length, allowing much higher pump power for each frequency components of the pulse signal before the saturation appears; also the varied Brillouin peak gives more uniform gain to each pulse spectral component than the pump modulation scheme. As long as the fiber is designed with appropriate frequency location, slope and varied Brillouin frequency covers the pulse spectrum. This method is very different from any pump modulation scheme in which the frequency resonance condition is the same over the entire fiber distance $[4,6]$, the same principle applies to resonance and absorption scheme [7], in which the pump source includes two frequencies at Stokes and anti-Stokes 
at $\omega_{0}+\Omega_{B}$ and $\omega_{0}-\Omega_{B}$ for the probe signal of $\omega_{0}$ to get zero gain through the combination of an absorption and gain resonance. However, the energy exchange between the $\omega_{0}+$ $\Omega_{B}$ as a pump signal and the pulsed signal $\omega_{0}$ is not the same as the energy exchange between the $\omega_{0}$ as a pulsed probe signal and the modulated signal of $\omega_{0}-\Omega_{B}$, as the pump energy is always much stronger than that of the probe signal. Therefore, the unbalance of the absorption and gain will be created along the fiber length and this process is nonunfiorm due to the higher depletion of the gain, especially when the long fiber length or higher pump power are used to achieve larger delay. While in nonuniform fiber the situation is different, because the variable Brillouin frequency locates at different fiber locations, the pump power is transferred to the different frequency components which are in resonance of the Brillouin frequency for the probe beam (data pulse). Therefore, the nonuniform fiber provides a solution of flexibility and simplicity for slow light generation with CW pump and no need for the complicated modulation form. This idea was first demonstrated theoretically in [8] with linear relation of the Brillouin frequency versus the fiber location, and Brillouin gain is the Lorentzian shape of $30 \mathrm{MHz}$. In this work, the Brillouin gain has double peaks with broader bandwidth of $500 \mathrm{MHz}$, the peak Brillouin frequency varies along the fiber within $500 \mathrm{MHz}$ to cover 3 nanoseconds pulse spectrum. This process provides higher power spectrum density over effectively short fiber length for the specific pulse spectral component of the data pulses and avoids the saturation of the peak gain of the Brillouin spectrum. This preserves high fidelity of the input pulse shape and allows high gain and large delay over the entire pulse spectrum and fiber length, thus the saturation impairment is reduced significantly, which leads to minimum distortion for the output pulse.

In the paper, we experimentally demonstrated long fractional delay of nanosecond data pulse with low distortion based on SBS slow light by using nonuniform dispersion decreasing fiber (DDF). With using a CW pump only, the scheme achieves a delay exceeding a pulse width at particular beat frequency and with larger delay versus gain slope coefficient and much better output pulse quality than single mode fiber.

\section{Experiment, Results, and Discussion}

The experimental configuration for observing slow light via SBS is similar to the setup in [9] for the pump and probe interaction in the Brillouin medium of DDF [10]. The wavelength of the probe and pumplight is $\sim 1319 \mathrm{~nm}$. The probe light $\nu_{S}$ is modulated by a pulse generator through an electro-optic modulator (EOM) to become a data pulse with 3 nanosecond pulse width and coupled into DDF from its high-dispersion side. The pump light $v_{P}$ is launched into the fiber from its low-dispersion side. The peak power of the data pulse is $2.3 \mathrm{~mW}$ and the pump power varies from 0 to $17 \mathrm{~mW}$ through an optical attenuator. By tuning the wavelength of the probe light, we can finely tune and lock the beat frequency (measured by frequency counter) between

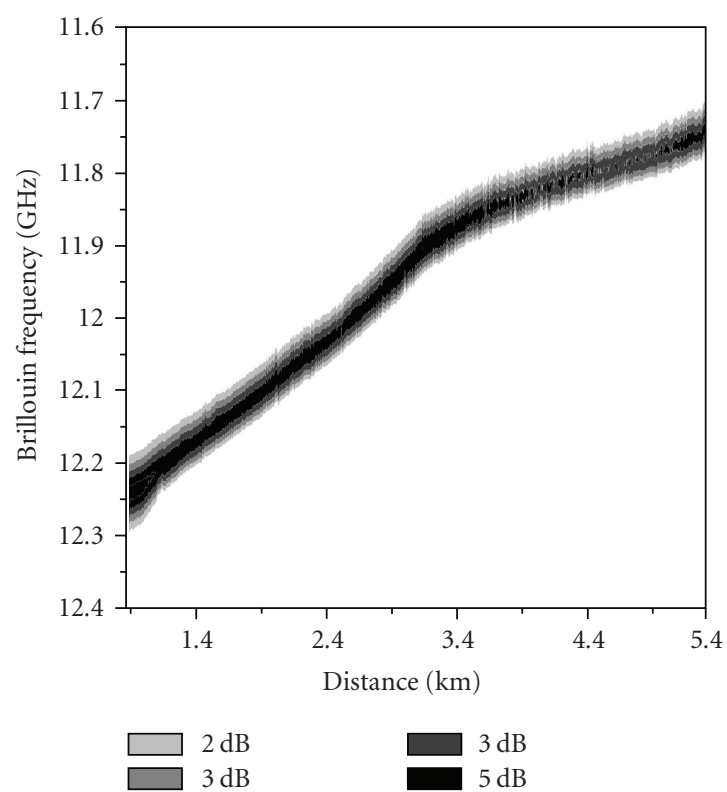

FIGURE 1: DDF Brillouin gain spectra along fiber length.

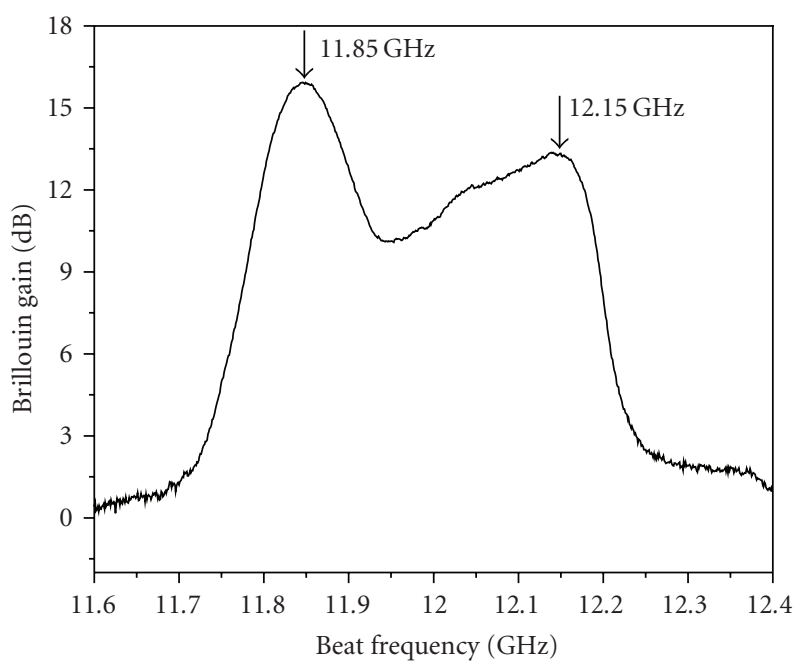

Figure 2: Brillouin gain spectrum of DDF.

the pump and probe $\left(\nu_{B}=v_{S}-v_{P}\right)$ within $\mathrm{Hz}$ using a phaselocked feedback circuit within frequency counter.

By measuring the depleted CW pump beam from the output of the circulator and scanning the beat frequency of the two lasers, a Brillouin loss spectrum of DDF from 11.6 to $12.4 \mathrm{GHz}$ is obtained as shown in Figure 1 . DDF has decreasing core refractive indices along the fiber and a dispersion parameter of 7.7 (high-dispersion side) and $-0.3 \mathrm{ps} / \mathrm{nm} / \mathrm{km}$ (low-dispersion side) at wavelength of $1550 \mathrm{~nm}$. The mode field diameter is about $5.0-7.0 \mu \mathrm{m}$ and the fiber loss is $0.45 \mathrm{~dB} / \mathrm{km}$. The length of DDF is $5.4 \mathrm{~km}$. The overall Brillouin gain spectrum of the DDF was also measured using the same method, as shown in Figure 2.

As can be seen, there are two peak frequencies in the gain spectrum profile: one is located at $11.85 \mathrm{GHz}$, which is the 


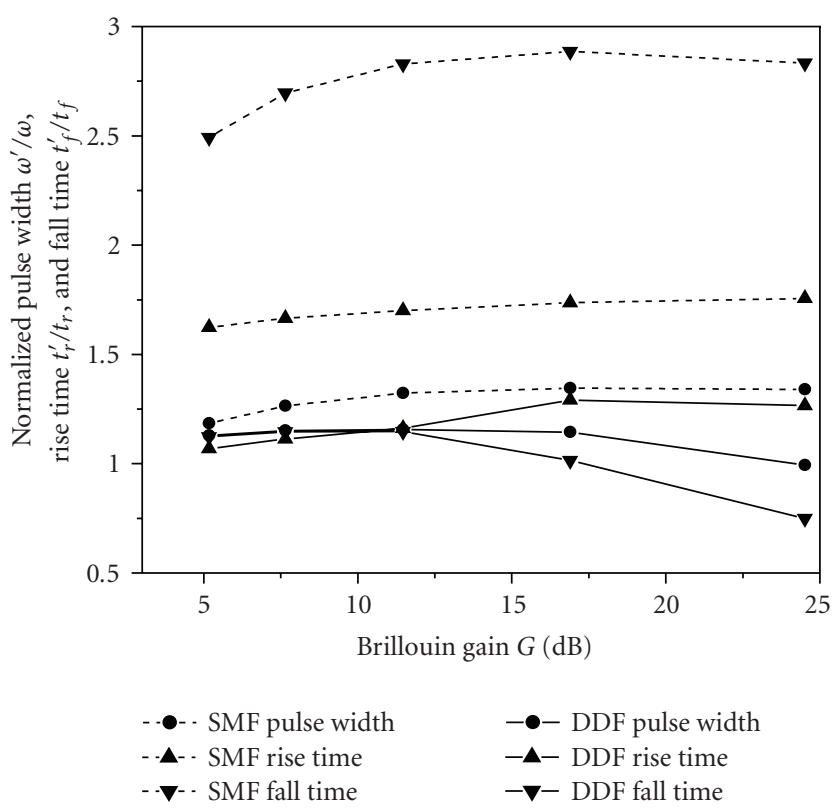

Figure 3: Normalized pulse width, rise, and fall times versus gain for DDF ( $w=3$ nanoseconds), comparing those for SMF ( $w=30$ nanoseconds).

Brillouin frequency around the midsection of the fiber as shown in Figure 1, and the other is at $12.15 \mathrm{GHz}$. This gain profile was designed to have varied chromatic dispersion to suppress the Brillouin threshold, which brings advantage of the smaller pulse fall time.

We measure the delay and data pulse distortion by analyzing oscilloscope records. The Brillouin gain $G$ is calculated by the ratio of the delayed data pulse intensity with nonzero pump power to the one without pump power. The delay $\tau$ is measured by the time difference between the peak time moments of the delayed pulses with and without pump power. Obviously, the larger values of these ratios indicate larger broadening and smoothing due to SBS, and hence the larger distortion of the data pulse. As the pump power changes from 0 to $17 \mathrm{~mW}$ for the fixed data pulse of $2.3 \mathrm{~mW}$, the Brillouin gain $G$ increases from 0 to $25 \mathrm{~dB}$, giving maximum delay $\tau=3.17$ nanseconds and relative delay $\tau / w=1.1$ bit. The normalized pulse width $\left(w^{\prime} / w\right)$, rise time $\left(t_{r}^{\prime} / t_{r}\right)$, and fall time $\left(t_{f}^{\prime} / t_{f}\right)$ is $1.0,1.27$, and 0.75 , respectively, while the normalized pulse width remains around 1 as shown in Figure 3 (solid), when the beat frequency is locked at $12.15 \mathrm{GHz}$ and the gain is $25 \mathrm{~dB}$. Here, $w^{\prime}, t_{r}^{\prime}$, and $t_{f}^{\prime}$ denote the pulse width, rise, and fall time of delayed data pulse, and $w, t_{r}$, and $t_{f}$ denote the pulse width, rise, and fall time of the data pulse, when the optical power of pump laser is zero. The SMF28 results of CW pumping of 30 nanosecond (for $30 \mathrm{MHz}$ bandwidth) pulse is shown in dotted line of the same figure as comparison.

The compensation effect of DDF can be explained as follows. When the beat frequency is locked at $12.15 \mathrm{GHz}$, the central frequency of the data pulse is located at the gain peak wavelength, while the lower-frequency components relative to peak frequency in the pulse spectrum are in the region

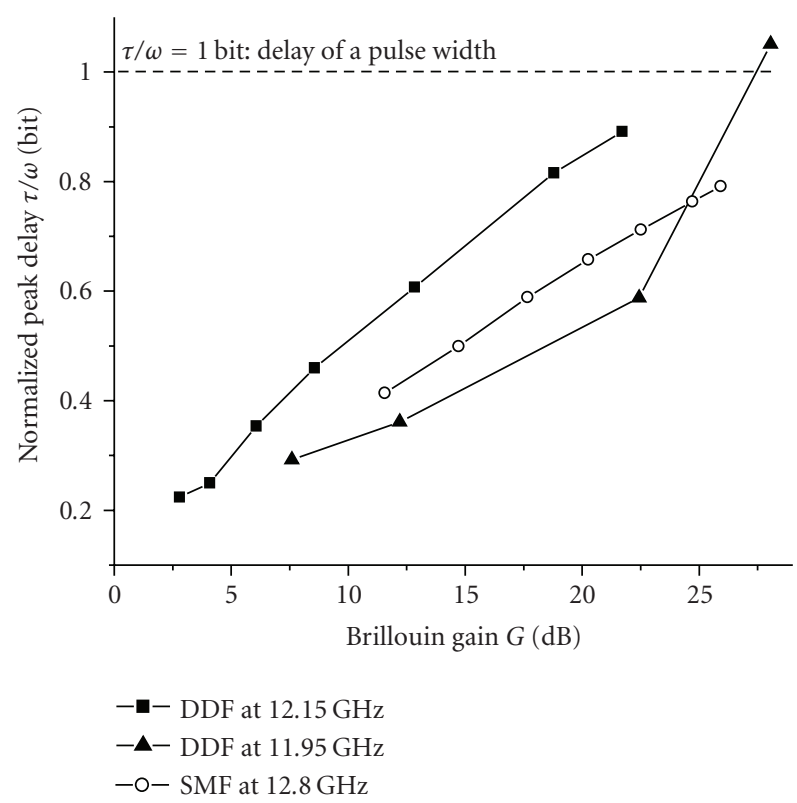

FIgURE 4: Normalized peak delays versus gain for DDF when the beat frequency is locked at 11.95 and $12.15 \mathrm{GHz}$, comparing to those for single-mode fiber at $12.8 \mathrm{GHz}$.

with low gain, as shown in Figure 2. The high gains of side frequency components of DDF broaden the bandwidth of the data pulse and compensate the pulse broadening, thus reduce the distortion. The smaller fall time in DDF is caused by the chromatic dispersion introduced by the dispersion decreasing fiber, as this fiber has a dispersion parameter of 7.7 (high-dispersion side) and $-0.3 \mathrm{ps} / \mathrm{nm} / \mathrm{km}$ (low-dispersion side). The probe pulse is launched at the high-dispersion end, and the SBS slow-light generation also creates the dispersion, this slow-light generated additional dispersion has been overcorrected by the fiber chromatic dispersion, therefore the fall time has been reduced.

Next, we tune and lock the beat frequency between probe and pump to $11.85,11.95,12.05$, and $12.15 \mathrm{GHz}$, respectively. We have found that at beat frequency of $12.15 \mathrm{GHz}$, the minimum pulse distortion can be achieved, as shown in Figure 3. The linear delay is due to the relative lower Brillouin gain of the second Brillouin peak in Figure 2. The maximum delay is found at $11.95 \mathrm{GHz}$ and the nonlinear relation is accounted from double peak Brillouin spectrum in Figure 2 in which $11.95 \mathrm{GHz}$ corresponds to center of Brillouin gain spectrum between two Brillouin peaks. Figure 4 shows the normalized delays versus gain for DDF when the beat frequency is locked at 11.95 and $12.15 \mathrm{GHz}$, respectively, as compared to those for single-mode fiber. One can see from the figure, when the gain is $28 \mathrm{~dB}$ and the beat frequency is $11.95 \mathrm{GHz}$, a maximum of 1.1 bit delay can be achieved for DDF. We also find that at different beat frequency, the normalized delay $\tau / w$ increases with different slope coefficients $\alpha$ as the gain increases (Table 1). It is notable that the coefficient for DDF at $12.15 \mathrm{GHz}$ is $0.036 \mathrm{bit} / \mathrm{dB}$ and larger than the coefficient for single-mode fiber of $0.026 \mathrm{bit} / \mathrm{dB}$. 
TABLE 1: The slope coefficients of normalized delay versus gain $\left(\alpha\right.$ in bit/dB) at different beat frequencies $\nu_{B}$.

\begin{tabular}{llllll}
\hline$\nu_{B}$ & 11.85 & 11.95 & 12.05 & 12.15 & $12.8(\mathrm{SMF})$ \\
\hline$\alpha$ & 0.026 & 0.034 & 0.018 & 0.036 & 0.026 \\
\hline
\end{tabular}

\section{Conclusions}

In summary, we have found that a maximum of $1.1 \mathrm{bit}$ relative delay can be achieved for $500 \mathrm{MHz}$ bandwidth nonuniform fiber and 3-nanosecond pulse with delay gain ratio of $0.036 \mathrm{bit} / \mathrm{dB}$. The smallest pulse distortion can be achieved when the beat frequency is locked at $12.15 \mathrm{GHz}$, which has a frequency offset to the center of the Brillouin spectrum profile, and the slope coefficient of the relative delay versus gain is larger than that of single-mode fiber. The current work has provided a guideline for making nonunfiorm fiber as slow light generator, the better designed fiber can be made to increase the range of the variable Brillouin frequency and to cover the 10 Gbps signal and in the same time to incorporate the chromatic dispersion compensation via variable chromatic dispersion along the fiber. This can generate ultimate simple solution for generation of the slow light via SBS using nonuniform fiber.

\section{Acknowledgments}

The authors would like to thank the Corning to provide DDF fiber. The research is supported by NSERC Agile All Photonics Networks, and NSERC Discovery grant.

\section{References}

[1] K. Y. Song, M. Herráez, and L. Thévenaz, "Observation of pulse delaying and advancement in optical fibers using stimulated Brillouin scattering," Optics Express, vol. 13, no. 1, pp. 82-88, 2005.

[2] Y. Okawachi, M. S. Bigelow, J. E. Sharping, et al., "Tunable all-optical delays via Brillouin slow light in an optical fiber," Physical Review Letters, vol. 94, no. 15, Article ID 153902, 4 pages, 2005.

[3] Z. Zhu, A. M. C. Dawes, D. J. Gauthier, L. Zhang, and A. E. Willner, "Broadband SBS slow light in an optical fiber," Journal of Lightwave Technology, vol. 25, no. 1, pp. 201-206, 2007.

[4] K. Y. Song and K. Hotate, "25 GHz bandwidth Brillouin slow light in optical fibers," Optics Letters, vol. 32, no. 3, pp. 217 219, 2007.

[5] V. P. Kalosha, L. Chen, and X. Bao, "Slow and fast light via SBS in optical fibers for short pulses and broadband pump," Optics Express, vol. 14, no. 26, pp. 12693-12703, 2006.

[6] Z. Lu, Y. Dong, and Q. Li, "Slow light in multi-line Brillouin gain spectrum," Optics Express, vol. 15, no. 4, pp. 1871-1877, 2007.

[7] R. Pant, M. D. Stenner, M. A. Neifeld, and D. J. Gauthier, "Optimal pump profile designs for broadband SBS slow-light systems,” Optics Express, vol. 16, no. 4, pp. 2764-2777, 2008.

[8] V. P. Kalosha, L. Chen, and X. Bao, "Slow light of subnanosecond pulses via stimulated Brillouin scattering in nonuniform fibers," Physical Review A, vol. 75, no. 2, Article ID 021802, 4 pages, 2007.
[9] L. Zou, X. Bao, Y. Wan, and L. Chen, "Coherent probe-pumpbased Brillouin sensor for centimeter-crack detection," Optics Letters, vol. 30, no. 4, pp. 370-372, 2005.

[10] M.-J. Li, S. Li, and D. A. Nolan, "Nonlinear fibers for signal processing using optical Kerr effects," Journal of Lightwave Technology, vol. 23, no. 11, pp. 3606-3614, 2005. 

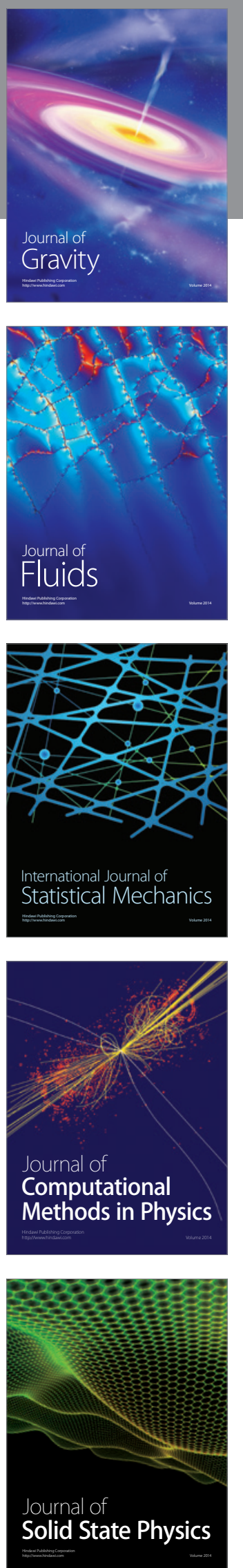

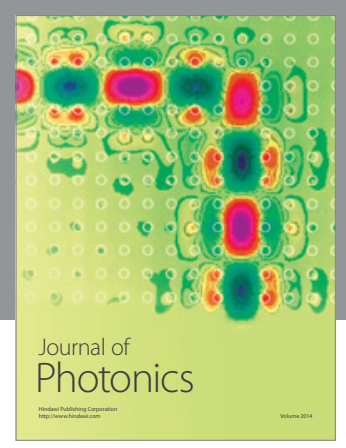

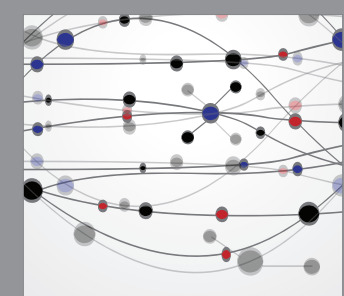

The Scientific World Journal
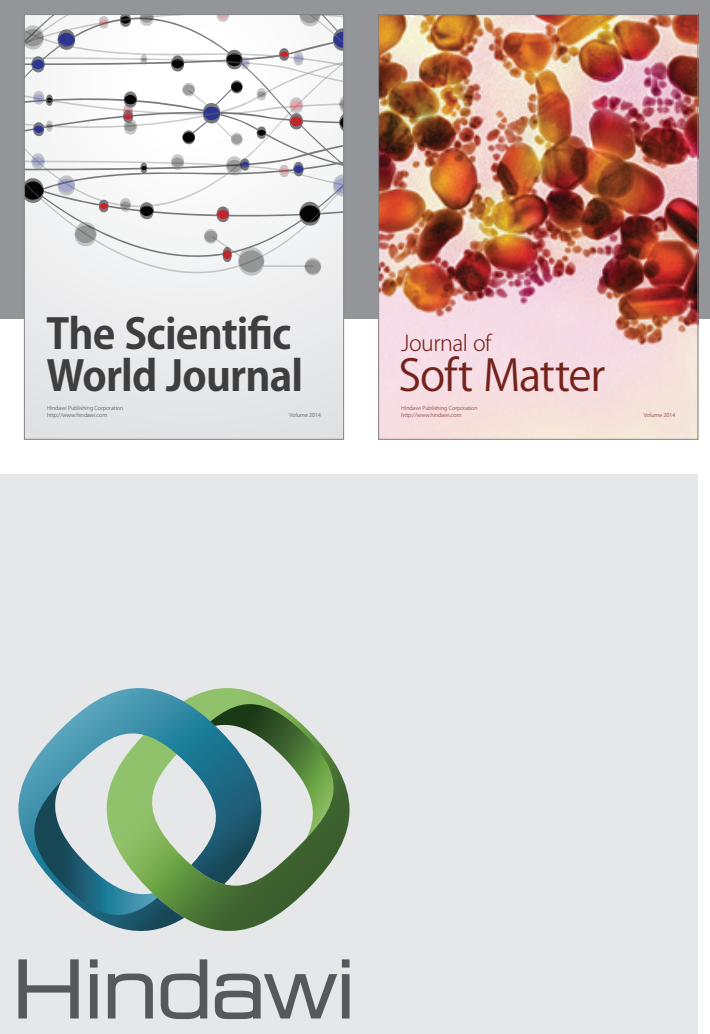

Submit your manuscripts at

http://www.hindawi.com
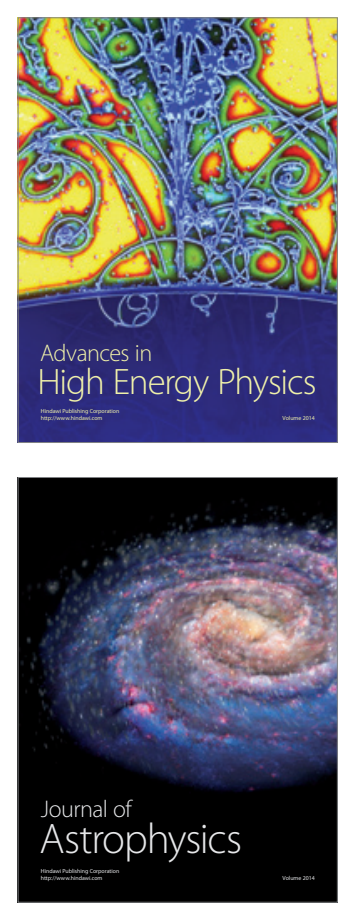
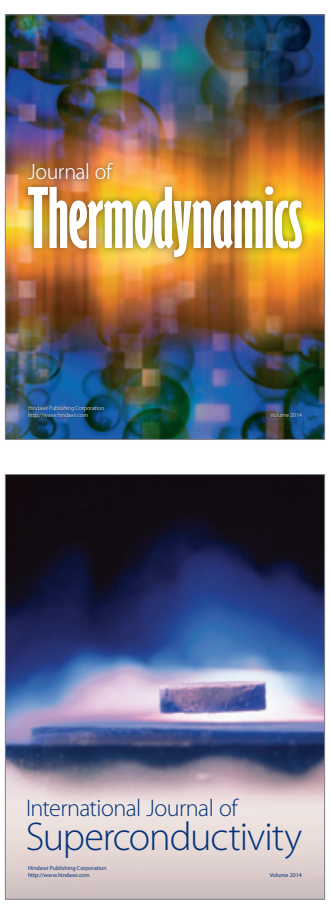
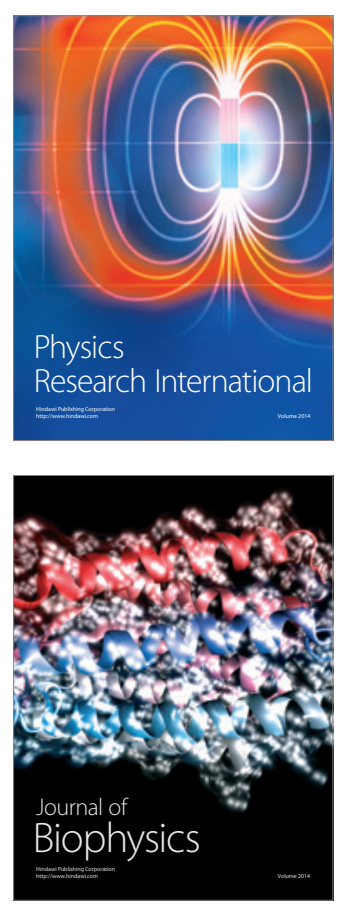
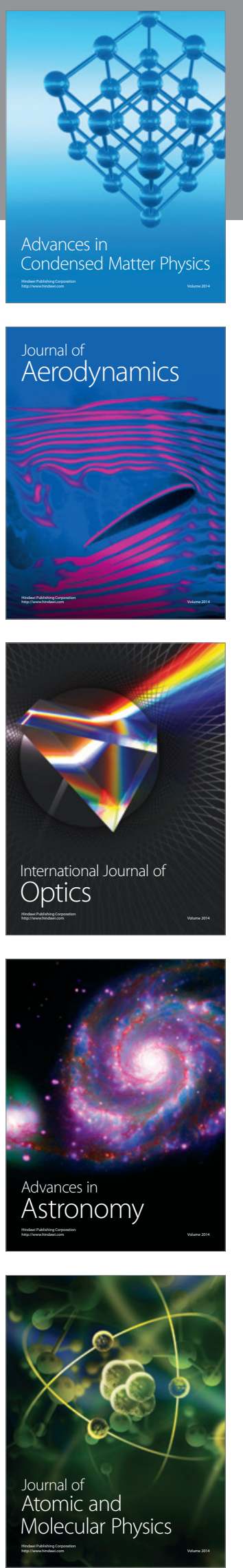